\title{
Provision of micronutrient-fortified food from 6 months of age does not permit HIV- exposed, uninfected Zambian children to catch up in growth to HIV-unexposed children: a randomised controlled trial
}

Suzanne Filteau, PhD, ${ }^{1}$ Kathy Baisley, MSc, ${ }^{1}$ Molly Chisenga, $\mathrm{RN},{ }^{2}$ Lackson Kasonka, MD, ${ }^{2}$ Rosalind S. Gibson, PhD, ${ }^{3}$ and the CIGNIS Study Team ${ }^{4}$

${ }^{1}$ London School of Hygiene and Tropical Medicine; ${ }^{2}$ University Teaching Hospital, Lusaka; ${ }^{3}$ Department of Human Nutrition, University of Otago, Dunedin, New Zealand

${ }^{4}$ The CIGNIS study team comprises: Principal Investigator: Suzanne Filteau, London School of Hygiene and Tropical Medicine (LSHTM) ; Zambian Lead Investigator: Lackson Kasonka, University Teaching Hospital (UTH), Lusaka; Senior Investigators: Rosalind Gibson, University of Otago, New Zealand; Ursula A. Gompels, LSHTM; Shabbar Jaffar, LSHTM; Emmanuel Kafwembe, Tropical Diseases Research Centre, Ndola; Mwaka Monze, UTH; Moses Sinkala, Catholic Relief Services, Zambia; Andrew Tomkins, Institute of Child Health, University College, London; Rodah Zulu, National Institute of Science and Industrial Research, Zambia; Clinic Coordinator: Molly Chisenga; Clinical Officer: Joshua Siame; Data Manager: Hildah Banda Mabuda; Statisticians: Kathy Baisley, Helen Dale, Andrea Rehman; Research Fellows: Matthew Bates, Anne Mullen; Clinic Staff: Hellen Kangwa Bwalya, Margaret Chileshe, Priscilla Kangwa Kowa, Mabvuto Kumwenda, Munalula Likando, Sydney Mambwe, Mutinta Muzyamba, Anne Mwale, Lungowe Nyaywa; Laboratory Staff: Mirriam Kapambwe, Humphrey Bima, Laura Gosset, Louise Hackett, Abigail Jackson, Eric Njunju, Sydney Mwanza; Data Entry: Natalia Shampwaya, Concillia Kabanga, Philip Soko; Drivers and Cleaners: John Chobo, Winford Kapumba, Charity Musonda

Corresponding author: Suzanne Filteau, Nutrition and Public Health Interventions Research Unit, London School of Hygiene and Tropical Medicine, Keppel Street, London WC1E 7HT, UK Telephone: (+44)-207-958-8108; fax: (+44)-207-958-8111; email: Suzanne.Filteau@lshtm.ac.uk

A preliminary version of the results was presented as a poster to the International Nutrition Congress, Bangkok, Thailand, October 2009

Sources of support: The project was funded by the Bill and Melinda Gates Foundation with micronutrients provided by DSM South Africa

Running head: HIV exposure and growth of Zambian children 


\section{Abstract}

Background: HIV-exposed, uninfected (HIV-EU) children represent a large proportion of children in southern Africa. The reasons for their poorer growth and higher morbidity and mortality than their HIV-unexposed peers are unclear.

Objective: We compared anthropometry of 125 HIV-EU and 382 HIV-unexposed young Zambian children participating in a trial of micronutrient-fortified complementary/replacement food.

Design: The randomised controlled trial provided children from age 6 to 18 months with a porridge flour containing either a basal or a rich level of micronutrients. Weight and length were measured 3-monthly and head and arm circumferences and triceps and subscapular skinfolds 6-monthly.

Results: There were no significant anthropometric differences between the two treatment groups. In unadjusted analyses, most anthropometric Z scores of HIV-EU children were lower than those of HIV-unexposed children; following adjustment for treatment arm, socioeconomic factors, breastfeeding and sex, head and arm circumference $Z$ scores remained lower.

Subscapular skinfold Z scores were lower among HIV-EU than HIV-unexposed children at 6 months but not 18 months.

Conclusions: Socioeconomic factors accounted for some but not all of the impaired growth of HIV-EU children. Micronutrient malnutrition may not be the socioeconomic factor responsible for the growth faltering. Factors acting earlier in life had irreversible effects.

Key words: growth, children, HIV-exposed, micronutrients 


\section{Introduction}

Children born to HIV-infected mothers who themselves escape HIV-infection can be described as HIV-exposed, uninfected (HIV-EU). HIV transmission rates among breastfed infants where either mother or infant is provided with antiretroviral therapy during breastfeeding, as recently recommended, ${ }^{1}$ can be less than $10 \% .{ }^{2}$ In the countries in Sub-Saharan Africa where antenatal HIV prevalence can be $20-30 \%,{ }^{3}$ HIV-EU children may therefore be up to a quarter of all children born. Therefore, any health or development problems they experience are of enormous public health importance.

Growth of HIV-EU children appears to be poorer and mortality greater than that of their HIVunexposed counterparts, ${ }^{4}$ although they do not do as badly as HIV-infected children. Growth faltering in childhood is a non-specific indicator of health problems and is associated with adverse effects in both the short ${ }^{5}$ and the long ${ }^{6}$ term. Although the causes of the poor growth and health of HIV-EU African children are not established, problems likely start early and are not limited to orphaned children. HIV-EU children are born with a slightly lower birth weight than unexposed children. ${ }^{7-8}$ Some studies have reported that they soon catch up in growth, 7,9 whereas others have not. ${ }^{10}$ Differences in breastfeeding practices and diets available in later infancy, as well as whether or not mortality of the most malnourished children was accounted for, may explain different findings. Other factors which may contribute to the health problems of HIV-EU children, as well as to the differences among studies, are increased exposure to infections and exposure to antiretroviral drugs. ${ }^{4}$

In the present study, we investigated whether provision of a nutritionally complete complementary or replacement food, compared with a food adequate in calories but of lower micronutrient density, from age 6 to 18 months would improve growth and permit HIV-EU children to catch up in growth with their HIV-unexposed peers.

\section{Methods}

\section{Study design}

The Chilenje Infant Growth, Nutrition and Infection Study (CIGNIS) trial is registered as ISRCTN37460449 (www.controlled-trials.com/mrct). Children were randomised to one of two locally produced porridge flours at age 6 months, and were followed monthly for 12 months. Detailed methods and results of the primary outcome (prevalence of stunting at 18 months) have been published. ${ }^{11}$

\section{Study population and recruitment}

The trial was conducted in Chilenje, Lusaka, Zambia, from October 2005 to July 2009. Infants were eligible if they were 6 months $+/-2$ weeks old, in generally good health, and their mothers gave written consent. Knowledge of maternal HIV status was not a requirement for joining the study but $90 \%$ of women knew their status from antenatal testing in the government health service. Since the focus of the present work was on HIV-EU children, only those infants whose mother's HIV status was known and who themselves tested HIV-negative at 18 months are included in the present analyses. 
Randomisation

Randomisation was in blocks of 20; the code was held by the project Data Safety and Monitoring Board (DSMB). Infants were allocated to porridges by sequential numbers on recruitment.

\section{Study porridges}

The two flours, based on maize, beans, bambaranuts and groundnuts, were similar in bulk ingredients and macronutrient content but differed in micronutrient content (Supplementary Table 1). In pilot work, porridges were found to be acceptable ${ }^{12}$ to the local population and to displace other micronutrient-poor complementary or family foods but not to displace breast milk intake. ${ }^{13}$ Both porridges contained recommended macronutrient content. ${ }^{14}$ The richly-fortified porridge contained micronutrients to meet the WHO estimated needs ${ }^{14}$ for infants aged 9-11 months with low breast milk intake, assuming an intake of $50 \mathrm{~g}$ flour/day. The basal porridge had micronutrients at levels which would have been in the maize component if a national maize fortification ${ }^{15-16}$ were introduced as previously planned. Micronutrient premixes for both flours were prepared by DSM (Isando, South Africa). Flour was mixed and processed by extrusion at Quality Commodities Ltd, Lusaka and stored for up to 6 months in a cool, well-ventilated room.

\section{Follow-up and anthropometry}

At recruitment, socio-demographic information was obtained by a pre-tested questionnaire. Women were given $4 \mathrm{~kg}$ of porridge flour each month and could return for more as required. At each monthly visit, women were asked how much porridge their child had consumed in the past 2 days, and if those days were typical. The mean and standard deviation (SD) reported intake of the porridge food provided was calculated for 'typical' days. Women were also asked if they were still breastfeeding, and, if so, approximately how many times a day they breastfed their child. If no longer breastfeeding, they were asked when they stopped.

Weight and length were measured every 3 months and other anthropometry every 6 months in the clinic. Anthropometric measurements were taken using calibrated equipment and standardized techniques, ${ }^{17}$ with children nude or wearing a diaper. Weight was measured on a digital balance (to $10 \mathrm{~g}$ ) and length on a length board to $1 \mathrm{~mm}$ (anthropometry equipment from Chasmor Ltd, London, UK). Head circumference and mid-upper arm circumference were measured using a fibre-glass insertion tape, and triceps and sub-scapular skinfolds using a precision Holtain skinfold caliper. Anthropometrists were trained using a repeat-measures protocol until they reached an acceptable level of proficiency ${ }^{18}$. Measurement quality was maintained by achieving acceptable ranges for both the differences in measurements between the designated criterion anthropometrist (MC) and the other anthropometrists ${ }^{18}$ and for the total technical error of each measurement. ${ }^{19}$

All anthropometric measurements were done in triplicate and the median used in analyses. Standard deviation, Z, scores were calculated using the World Health Organization (WHO) growth reference data. ${ }^{20}$

\section{HIV testing}

Serum samples from all children at 18 months were tested for antibodies to HIV. A serial testing algorithm was used. Samples were first tested using Determine HIV $1 / 2$ (Inverness Medical, Japan). If negative, the result is taken to be negative. If positive, a second test, Unigold HIV 1/ 2 (Trinity Biotech plc, Ireland), was used. If positive, the final result is positive but if negative, i.e. discordant, a third test SD-Bioline HIV $1 / 2$ (Standard Diagnostics, Korea) was used and its result was taken to be the final result. 
Statistical analyses

The analysis for this study was restricted to children who completed at 18 months, were known HIV negative, and whose mothers' antenatal HIV status was known. All analyses of the effect of diet group were described in an analysis plan that was approved before the study was unblinded.

Characteristics between maternal HIV groups at baseline were compared using Chi-squared tests for categorical variables and t-test for continuous variables. Since the randomisation was not stratified by maternal HIV status, we also compared diet groups within each maternal HIV group. Lastly, within each maternal HIV group, we compared baseline characteristics of those who were included in the present study and those who were not.

To assess the effect of the fortified diet on child growth over time, data from the 6-18 month visits were combined, and mean anthropometric Z-scores were compared between diet groups. Random effects regression was used to account for the correlation of repeated measurements within children. Analyses were stratified by maternal HIV. Initial models contained fixed effects for time (visit), and a random intercept for each child. Visit was fit as a linear and a quadratic term, unless the likelihood ratio test for departure from linearity was not significant at $p<0.05$. If the quadratic term was not adequate, visit was fit as a categorical factor. Secondary analyses were adjusted for a priori confounders selected because of their known associations with child growth: socioeconomic status (SES), maternal education, current breastfeeding, child sex, and baseline ( 6 month) Z-score (to control for possible growth deficits before the child was given the diet) as a continuous covariate. SES was measured using an asset index, created by combining data on possessions and housing characteristics using principal component analysis. ${ }^{21}$ Within each maternal HIV group, changes in Z-scores over time (increase vs decrease) were evaluated by the estimate of the association with visit.

To assess the effect of maternal HIV on child growth over time, mean anthropometric Z-scores were compared between children of HIV-negative and HIV-positive mothers, using random effects regression as described above. Initial models contained fixed effects for diet group and visit, and a random intercept for each child. Adjusted analyses included effects for diet group, visit, SES, maternal education, current breastfeeding, and child sex. A test for interaction between maternal HIV status and visit was done to assess whether the effect of maternal HIV varied over time. We did not adjust for baseline $Z$ scores in this analysis, since baseline $Z$ scores were on the causal pathway between our exposure of interest (maternal HIV status) and outcome (growth over time).

To assess the effect of continued breastfeeding on growth over time, mean anthropometric Zscores were compared between children who were currently breastfeeding at the time of measurement and those who were not, using random effects regression as described above. Adjusted analyses included effects for diet group, time, SES, maternal education, child sex and baseline Z-score.

\section{Sample size}

Sample size calculations were based on the primary outcome of stunting at 18 months, assuming a prevalence of $40 \%{ }^{22}$ among children receiving the basal porridge. With a $15 \%$ loss to follow up, 400 children per group would provide $>90 \%$ power to detect a $30 \%$ reduction in the prevalence of stunting, and $>80 \%$ power to detect a $25 \%$ reduction (alpha $=0.05$ ). The DSMB reviewed the results twice (September 2007 and May 2008) and, after noting the prevalence of stunting was lower than anticipated, and that an increase in sample size was financially and logistically impossible, recommended stopping recruitment in mid-July 2008. 
Ethics

The study was approved by the ethics committees of the University of Zambia and the London School of Hygiene and Tropical Medicine. Mothers gave written informed consent. Project staff referred participants who were ill to local medical services and followed up treatments within these services.

\section{Results}

A total of 743 infants were recruited and randomised but the present analysis is restricted to the 507 children whose mothers' antenatal HIV status was known and who were themselves HIVuninfected (Figure 1). In this sub-group of children, as in the trial cohort overall, ${ }^{11}$ treatment groups were similar at baseline except there was a slight excess of girls in the richly-fortified diet group (Table 1). In addition, among HIV-EU children, those in the richly-fortified group had slightly lower mean weight at 6 months and lower maternal education than those in the basal arm. HIV-positive mothers were older and of lower education and SES than HIV-negative mothers, and all anthropometric indices at 6 months were significantly lower in HIV-EU children. Most strikingly, whereas virtually all HIV-uninfected women were still breastfeeding, a large proportion of HIV-infected women either never initiated breastfeeding or else had stopped before 6 months.

Mothers who were included in the present study were older and more likely to be married than those who were not included $(p<0.05)$, and HIV-negative mothers who were included had higher education than those not included. There were no differences in any of the baseline anthropometric indices or other characteristics of children who were included and those who were not.

Neither the number of visits attended nor porridge consumption differed between treatment groups (data not shown). The median daily amount of porridge consumed increased with age from $\sim 275 \mathrm{ml} / \mathrm{d}(\sim 19 \mathrm{~g}$ flour $/ \mathrm{d})$ at 7 months to $\sim 400 \mathrm{ml} / \mathrm{d}(\sim 28 \mathrm{~g}$ flour $/ \mathrm{d})$ from 11 to 18 months. These amounts were only about half the estimated consumption used as the basis for the micronutrient concentrations in the richly-fortified diet. Consumption of the study diet was significantly greater among children of HIV-infected women, women who were not breastfeeding, and those with lower socioeconomic status. At 7 months, median daily porridge consumption among children who were breastfeeding was $250 \mathrm{ml} / \mathrm{d}$, versus $400 \mathrm{ml} / \mathrm{d}$ among children who were not breastfeeding. From 11-18 months, median daily porridge consumption was $400 \mathrm{ml} / \mathrm{d}$ among children who were breastfeeding, and $450 \mathrm{ml} / \mathrm{d}$ among those who were not breastfeeding.

Between ages 6 to 18 months, there were significant decreases in length, weight, head and arm circumference $Z$ scores and increases in triceps and subscapular skinfold $Z$ scores over time, among both HIV-EU and HIV-unexposed children (Figure 2). In the unadjusted analyses, diet treatment had no significant effect on the mean Z scores of HIV-EU or HIV-unexposed children (Table 2). However, after adjusting for a priori confounders, there was some evidence among children of HIV-negative mothers that mean head circumference-for age (adjusted mean difference $0.06(95 \% \mathrm{Cl}-0.003,0.11), \mathrm{P}=0.06)$ and weight-for-age (adjusted mean difference $0.08(95 \% \mathrm{Cl}-0.01,0.17), \mathrm{P}=0.10) \mathrm{Z}$-scores were higher in the micronutrient-fortified arm.

For all anthropometric variables, in the unadjusted analysis mean Z scores of HIV-EU children were significantly lower than those of HIV-unexposed children (Table 2). After adjustment for 
treatment arm, socioeconomic status, maternal education, current breastfeeding and child sex, mean head and arm circumference $Z$ scores remained significantly lower in the HIV-EU compared with the HIV-unexposed children. For subscapular skinfold Z scores, there was a significant interaction $(\mathrm{P}=0.003)$ between maternal HIV status and visit month, indicating that the effect of maternal HIV status on growth changed over time. At the 6 and 12 month visits, mean subscapular skinfold Z scores were significantly lower among HIV-EU children (mean difference at $6 \mathrm{~m}=-0.45(95 \% \mathrm{Cl}-0.67$ to -0.23$)$; at $12 \mathrm{~m}=-0.27(95 \% \mathrm{Cl}-0.50$ to -0.05$))$, but at 18 months, there was no significant difference between maternal HIV groups in mean subscapular skinfold $Z$ scores (mean difference $-0.15(95 \% \mathrm{Cl}-0.37$ to 0.07$)$; Figure 2 ).

Among both HIV-EU and HIV-unexposed children, there was evidence that the effect of breastfeeding on length, weight, and subscapular skinfold Z scores changed over time, with children who were still breastfeeding at later visits having lower mean Z scores than those who were no longer breastfeeding (Table 3). There was no evidence of an association with breastfeeding and head or arm circumference $Z$ scores, or triceps skinfold, in children of either maternal HIV group.

\section{Discussion}

The CIGNIS study aimed to improve health and growth of young Zambian children by providing high quality complementary or replacement foods from 6 to 18 months of age, a period with a high risk of growth faltering. ${ }^{23}$ Although intake of the diets was only half of the expected when we planned fortificant levels, we have previously shown that the richly-fortified diet improved hemoglobin and iron status of all children and reduced stunting at 18 months among nonbreastfed children of HIV-infected mothers. ${ }^{11}$ In the present work the richly-fortified diet slightly increased weight and head circumference of the HIV-unexposed children but did not benefit growth of the HIV-EU children. For ethical reasons we provided food and medical care to all children and thus decreased the chance of detecting benefits from the richly-fortified compared with the basal diet. The overall stunting rate at 18 months of $20 \%$ was only half the $40 \%$ on which we based sample size calculations and lower than the $36 \%$ stunting we measured among Chilenje clinic children aged 18 months who were not part of the CIGNIS study because they reached 6 months before recruitment began. ${ }^{11}$ Other anthropometric $Z$ scores of these nonstudy children from the community were similarly slightly lower than among study children (data not shown).

Provision of nutritional complementary foods from 6 months of age was unable to reverse the growth deficits seen among the HIV-EU children compared with HIV-unexposed children. There has been relatively little research into growth of HIV-EU African children. Early research suggested the growth faltering compared to HIV-unexposed children was transient but our previous ${ }^{10}$ and current studies in Lusaka suggest some chronic and persistent growth impairments. There are many differences between the cohorts studied in the 1990s and the CIGNIS cohort. First, since our study was designed to investigate growth of HIV-EU children, we have more detailed anthropometric data than do previous studies. Second, the mothers and infants in our present and previous cohorts had access to perinatal nevirapine which prevents HIV infection among many high risk infants who are now part of the HIV-EU group. Third, in our study breastfeeding was not universal and was of limited duration. Fourth, in our study Z scores were calculated based on the WHO 2006 reference which crosses over the old NCHS/WHO growth reference within the age group we studied and thus complicates comparisons between studies. Our cohort represents HIV-affected women and infants in much of current urban Africa but the situation may soon change again as the new WHO HIV and infant feeding 
recommendations ${ }^{1}$ are rolled out. These recommend up to a year breastfeeding while women or infants are given antiretroviral drugs and should thus both increase breastfeeding rates and, by decreasing HIV transmission, increase the proportion of HIV-EU children.

Differences in anthropometric Z scores between HIV-EU and HIV-unexposed children were all statistically significant before adjustment for confounders and were about 0.3 to 0.4 , depending on the index, which are large enough to be a concern on a population basis. Furthermore, at enrolment, all anthropometric measures were significantly lower among HIV-EU children. Since we recruited only relatively healthy children aged 6 months, it is possible that the children most at risk of growth faltering, because of being born low birth weight, having a mother with advanced HIV, or succumbing to illness because of early lack of breastfeeding, had died before becoming eligible for our study. Therefore, we believe our results may underestimate the growth deficit of HIV-EU children.

Adjustment for breastfeeding and socioeconomic factors, that is, maternal education and tertile of asset index score, decreased, but did not eliminate, the differences in Z scores seen between HIV-EU and HIV-unexposed children. For all anthropometric measures, there was still some evidence of a negative effect after adjustment, although not always statistically significant. Furthermore, since few HIV-EU children were still breastfeeding at 6 months, the adjustment for breastfeeding was not very efficient, so we may have underestimated the effect of maternal HIV. The lower socioeconomic status of the HIV-infected women may contribute to the poorer growth of their children. Within high HIV prevalence African countries, socioeconomic factors have less association with HIV acquisition than in Western countries. ${ }^{24}$ Lower socioeconomic status, on the other hand, may result from HIV infection of working-age adults since it can impair their ability to earn money. Programmes such as food aid for HIV-exposed families may mitigate some of the effects of poverty. However, the present study indicates that provision of food to HIV-EU children from 6 months is too late to reverse the growth faltering which was present since birth. It remains to be seen whether provision of food aid to pregnant HIV-infected women could increase birth weight of their infants and reduce subsequent growth faltering. Provision of multivitamins to HIV-infected Tanzanian women increased weight at birth ${ }^{25}$ and at 24 months $^{26}$ of HIV-EU children.

The decrease between 6 and 18 months in length-for-age Z scores of even the HIV-unexposed children in this fairly middle income African population given free food suggests that nutrition after 6 months is not their main cause of growth faltering but other factors, such as exposure to infections, may be more important. Compared with HIV-unexposed children, HIV-EU children may be exposed to more infections because of their sick parents and may also be less resistant to infections, as indicated by their elevated mortality. ${ }^{4}$ We have shown higher rates of hospital referral among HIV-exposed versus unexposed children in the CIGNIS study. ${ }^{11}$

Subscapular skinfolds of the HIV-EU children approached those of the HIV-unexposed children between 6 and 18 months. It is debatable whether this growth in central fat is beneficial since early rapid increases in body fat have been associated amongst non-HIV-exposed children with later risk of chronic disease. ${ }^{27-28}$ Deposition of this fat centrally, rather than at peripheral sites, may be a response to stresses, such as exposure to maternal HIV in utero or during lactation. The consequences of deposition of increased central fat in this population, especially when exposed to the nutrition transition currently experienced by urban Zambia based on its rapidly increasing rates of overweight and obesity, ${ }^{29}$ remain unknown. It will be important to follow up these children to determine whether they develop risk factors for chronic disease and, if so, whether these can be ameliorated through lifestyle interventions. 
The effect of breastfeeding on anthropometry appeared to change with time, significantly for weight, length and subscapular skinfolds of both HIV-EU children and HIV-unexposed children. The trend towards lower $Z$ scores among children breastfed after 12 months may represent reverse causality, that is, mothers continued to breastfeed children who were growing poorly. The 12 month time point suggested by $\mathrm{WHO}^{1}$ as time to stop breastfeeding HIV-EU children was largely speculative but our results suggest it is reasonable in a population such as ours with access to clean water and adequate food.

A limitation of our study is the restriction to children who completed at 18 months, since PCR was not routinely available to test children for HIV at younger ages. However, there were no differences in most maternal characteristics, or in any anthropometric indices at 6 months, between children who were included and those excluded, so we believe our results to be generalisable.

Provision of nutritional complementary food was unable to reverse growth faltering among HIVEU children. Growth faltering is known to result from both poor nutrition and increased infection and our results suggest that nutrition and infection in the mother as well as the child need to be considered in health programs. In general, it is better to prevent growth faltering than to try to remedy it. Micronutrient supplementation of HIV-infected women should be explored further, based on its beneficial effects in Tanzania. ${ }^{25-26}$ Many African countries are now instituting programmes which provide antiretroviral therapy to women in late pregnancy, not just to mothers and their infants at delivery. With the new WHO guidelines, these antiretrovirals may soon be extended through lactation. It will be important to monitor whether these programmes reduce the growth and health problems of HIV-EU children.

\section{Acknowledgments}

We thank the entire CIGNIS study team and the participants. We are grateful to Dr. Bianca de Stavola, Reader in Biostatistics at the London School of Hygiene and Tropical Medicine, for statistical advice and to Mr. Chongo of Quality Commodities, Lusaka, for preparing and storing the flours. We are particularly grateful to the mothers and children who participated in the study and to the staff of Lusaka District Health who permitted and supported the study. The study was funded by the Bill and Melinda Gates Foundation with micronutrient premixes prepared and supplied by DSM, South Africa. 


\section{References}

1. World Health Organization (2009) New WHO recommendations on infant feeding in the context of HIV. Volume,

2. Becquet, R., et al., Universal antiretroviral therapy for pregnant and breast-feeding HIV1-infected women: towards the elimination of mother-to-child transmission of HIV-1 in resource-limited settings. Clin Infect Dis, 2009. 49(12): p. 1936-45.

3. World Health Organization. Online statistics. 2009 [cited; Available from: http://apps.who.int/globalatlas/default.asp.

4. $\quad$ Filteau, S., The HIV-exposed, uninfected African child. Trop Med Int Health, 2009. 14: p. 276-287.

5. $\quad$ Black, R.E., S.S. Morris, and J. Bryce, Where and why are 10 million children dying every year? Lancet, 2003. 361(9376): p. 2226-34.

6. Grantham-McGregor, S., et al., Developmental potential in the first 5 years for children in developing countries. Lancet, 2007. 369(9555): p. 60-70.

7. Bailey, R.C., et al., Growth of children according to maternal and child HIV, immunological and disease characteristics: a prospective cohort study in Kinshasa, Democratic Republic of Congo. Int J Epidemiol, 1999. 28(3): p. 532-40.

8. Marinda, E., et al., Child mortality according to maternal and infant HIV status in Zimbabwe. Pediatr Infect Dis J, 2007. 26(6): p. 519-26.

9. Lepage, P., et al., Growth of human immunodeficiency type 1-infected and uninfected children: a prospective cohort study in Kigali, Rwanda, 1988 to 1993. Pediatr Infect Dis J, 1996. 15(6): p. 479-85.

10. Makasa, M., et al., Early growth of infants of HIV-infected and uninfected Zambian women. Trop Med Int Health, 2007. 12(5): p. 594-602.

11. Chilenje Infant Growth Nutrition and Infection (CIGNIS) Study Team, Micronutrient fortification to improve growth and health of maternally HIV-unexposed and exposed Zambian infants: a randomised controlled trial PLoS One, 2010. http://www.plosone.org/article/info\%3Adoi\%2F10.1371\%2Fjournal.pone.0011165.

12. Owino, V., et al., Acceptability, storage stability and costing of alpha-amylase-treated maize-beans-groundnuts-bambaranuts complementary blend. J Sci Food Agriculture, 2007. 87: p. 1021-1029.

13. Owino, V., et al., Fortified complementary foods, with or without $\alpha$-amylase-treatment, increase hemoglobin but do not reduce breast milk intake of 9-month old Zambian infants. Am J Clin Nutr, 2007. 86: p. 1094-1103.

14. Dewey, K. and K. Brown, Update on technical issues concerning complementary feeding of young children in developing countries and implications for intervention programs. Special issue based on a World Health Organization expert consultation on complementary feeding. Food Nutr Bull, 2003. 24: p. 5-28.

15. Famine Early Warning Systems Network. Zambia Food Security Update. 2006 [cited; Available from: www.fews.net/docs/Publications/Zambia 200608en.pdf.

16. MOST (2005) Fortification Basics: maize flour/meal. Volume,

17. Lohman, T., A. Roche, and R. Martorell, Anthropometric standardization reference manual. 1988, Champaign, USA: Human Kinetics Publications.

18. Zerfas, A., Checking continuous measures: Manual for Anthropometry. 1985, Los Angeles, USA: Division of epidemiology, School of Public Health, University of California.

19. Ulijaszek, S. and D. Kerr, Anthropometric measurement error and the assessment of nutritional status. Br J Nutr, 1999. 82: p. 165-177.

20. World Health Organization (2006) Child growth standards. Volume, 
21. Filmer, D. and L. Pritchett, Estimating wealth effects without expenditure data - or tears: an application to educational enrolments in states of India. Demography, 2001. 38: p. 115-132.

22. World Health Organization (2002) Complementary feeding: Summary of guiding principles for complementary feeding of the breastfed child. Volume,

23. Shrimpton, R., et al., Worldwide timing of growth faltering: implications for nutritional interventions Pediatrics, 2001. 107: p. e75.

24. De Cock, K., D. Mbori-Ngacha, and E. Marum, Shadow on the continent: public health and HIVIAIDS in Africa in the 21st century. Lancet, 2002. 360: p. 67-72.

25. Fawzi, W.W., et al., Randomized trial of vitamin supplements in relation to vertical transmission of HIV-1 in Tanzania. J Acquir Immune Defic Syndr, 2000. 23(3): p. 246-54.

26. Villamor, E., et al., Vitamin supplementation of HIV-infected women improves postnatal child growth. Am J Clin Nutr, 2005. 81(4): p. 880-8.

27. Ekelund, U., et al., Upward weight percentile crossing in infancy and early childhood independently predicts fat mass in young adults: the Stockholm Weight Development Study (SWEDES). Am J Clin Nutr, 2006. 83(2): p. 324-30.

28. Ekelund, U., et al., Association of weight gain in infancy and early childhood with metabolic risk in young adults. J Clin Endocrinol Metab, 2007. 92(1): p. 98-103.

29. Zambian Central Statistical Office, et al., Zambia Demographic and Health Survey 2007. 2009, CSO and Macro International Inc: Calveton, Maryland, USA.

30. International Zinc Nutrition Consultative Group, Assessment of the Risk of Zinc Deficiency in Populations and Options for its Control. . Food Nutr Bull, 2004. 25: p. 94S203 S. 
Table 1. Baseline characteristics of 507 HIV-negative children completing the trial and of known maternal HIV status

\begin{tabular}{|c|c|c|c|c|c|c|}
\hline \multirow[b]{2}{*}{ Diet treatment group ${ }^{1}$} & \multicolumn{3}{|c|}{ HIV-unexposed children } & \multicolumn{3}{|c|}{ HIV-exposed, uninfected children } \\
\hline & Basal & $\begin{array}{l}\text { Richly- } \\
\text { fortified }\end{array}$ & $\begin{array}{c}\text { All HIV- } \\
\text { unexposed }\end{array}$ & Basal & $\begin{array}{l}\text { Richly- } \\
\text { fortified }\end{array}$ & $\begin{array}{c}\text { All HIV- } \\
\text { exposed } \\
\text { uninfected }^{2}\end{array}$ \\
\hline $\mathbf{N}$ & 190 & 192 & 382 & 66 & 59 & 125 \\
\hline Mother's age (years) & $25.8(\mathrm{SD} 5.8)$ & 26.1 (SD 5.8) & $25.9(\mathrm{SD}=5.8)$ & 28.3 (SD 5.3) & $29.4($ SD 5.4) & $28.8(\mathrm{SD}=5.4)$ \\
\hline \multicolumn{7}{|l|}{$\begin{array}{l}\text { Maternal body mass } \\
\text { index }\left(\mathrm{kg} / \mathrm{m}^{2}\right)\end{array}$} \\
\hline$<18.5$ & $13(7 \%)$ & $17(9 \%)$ & $30(8 \%)$ & $6(9 \%)$ & $5(8 \%)$ & $11(9 \%)$ \\
\hline $18.5-25$ & $115(61 \%)$ & $105(55 \%)$ & $220(58 \%)$ & $34(52 \%)$ & $36(61 \%)$ & $70(56 \%)$ \\
\hline $25-30$ & $42(22 \%)$ & $47(24 \%)$ & $89(23 \%)$ & $21(32 \%)$ & $12(20 \%)$ & $33(26 \%)$ \\
\hline$>30$ & $20(11 \%)$ & $23(12 \%)$ & $43(11 \%)$ & $5(8 \%)$ & $6(10 \%)$ & $11(9 \%)$ \\
\hline \multicolumn{7}{|l|}{ Mother's education } \\
\hline primary or less & $58(31 \%)$ & $48(25 \%)$ & $106(28 \%)$ & $20(30 \%)$ & $32(54 \%)$ & $52(42 \%)$ \\
\hline secondary & $77(41 \%)$ & $76(40 \%)$ & $153(40 \%)$ & $31(47 \%)$ & $16(27 \%)$ & $47(38 \%)$ \\
\hline college/university & $55(29 \%)$ & $68(35 \%)$ & $123(32 \%)$ & $15(23 \%)$ & $11(19 \%)$ & $26(21 \%)$ \\
\hline \multicolumn{7}{|l|}{ Mother's occupation } \\
\hline housewife & $104(55 \%)$ & $93(48 \%)$ & $197(52 \%)$ & $41(62 \%)$ & $35(59 \%)$ & $76(61 \%)$ \\
\hline salaried employee & $39(21 \%)$ & $45(23 \%)$ & $84(22 \%)$ & $9(14 \%)$ & $14(23 \%)$ & $23(18 \%)$ \\
\hline self-employed & $18(9 \%)$ & $16(8 \%)$ & $34(9 \%)$ & $10(15 \%)$ & $4(7 \%)$ & $14(11 \%)$ \\
\hline other & $29(15 \%)$ & $38(20 \%)$ & 67 (18\%) & $6(9 \%)$ & $6(10 \%)$ & $12(10 \%)$ \\
\hline \multicolumn{7}{|l|}{ Marital status } \\
\hline married & $144(76 \%)$ & $153(80 \%)$ & 297 (78\%) & $52(79 \%)$ & $46(78 \%)$ & $98(78 \%)$ \\
\hline single & $37(19 \%)$ & $35(18 \%)$ & $72(19 \%)$ & $10(15 \%)$ & $7(12 \%)$ & $17(14 \%)$ \\
\hline divorced, separated, & $9(5 \%)$ & $4(2 \%)$ & $13(3 \%)$ & $4(6 \%)$ & $6(10 \%)$ & $10(8 \%)$ \\
\hline \multicolumn{7}{|l|}{$\begin{array}{l}\text { Tertiles of } \\
\text { socioeconomic status }\end{array}$} \\
\hline low & $55(29 \%)$ & $59(31 \%)$ & $114(30 \%)$ & $25(38 \%)$ & $28(47 \%)$ & $53(42 \%)$ \\
\hline middle & 79 (42\%) & 74 (39\%) & $153(40 \%)$ & 25 (38\%) & 17 (29\%) & $42(34 \%)$ \\
\hline high & $56(29 \%)$ & $59(31 \%)$ & $115(30 \%)$ & $16(24 \%)$ & $14(24 \%)$ & $30(24 \%)$ \\
\hline
\end{tabular}




\begin{tabular}{|c|c|c|c|c|c|c|}
\hline \multicolumn{7}{|l|}{ Infant characteristics } \\
\hline $\operatorname{Sex}(\#, \% F)$ & $86(45 \%)$ & $102(53 \%)$ & $188(49 \%)$ & $34(52 \%)$ & $36(61 \%)$ & $70(56 \%)$ \\
\hline Birth weight (kg) & 3.06 (SD 0.54) & 3.08 (SD 0.47) & 3.07 (SD 0.50) & 3.04 (SD 0.46) & 2.93 (SD 0.52) & 2.99 (SD 0.49) \\
\hline \multicolumn{7}{|l|}{ Anthropometry at 6 months } \\
\hline Weight (kg) & 7.36 (SD 0.99) & 7.40 (SD 1.12) & 7.38 (SD 1.06) & 7.20 (SD 1.04) & 6.88 (SD 1.08) & 7.05 (SD 1.07) \\
\hline Length (cm) & 65.1 (SD 2.3) & 65.0 (SD 2.8) & 65.1 (SD 2.5) & 64.6 (SD 2.4) & 63.9 (SD 2.6) & $64.3(\mathrm{SD} 2.5)$ \\
\hline $\begin{array}{l}\text { Head circumference } \\
(\mathrm{cm})\end{array}$ & 43.7 (SD 1.4) & 43.7 (SD 1.4) & 43.7 (SD 1.4) & 43.3 (SD 1.3) & 43.1 (SD 1.5) & 43.2 (SD 1.4) \\
\hline Arm circumference $(\mathrm{cm})$ & 14.5 (SD 1.2) & 14.6 (SD 1.3) & 14.6 (SD 1.3) & 14.1 (SD 1.3) & 13.8 (SD 1.4) & 14.0 (SD 1.3) \\
\hline Triceps skinfold (mm) & 9.1 (SD 1.7) & 9.3 (SD 1.8) & 9.2 (SD 1.8) & 8.8 (SD 2.0) & 8.9 (SD 1.7) & 8.8 (SD 1.8) \\
\hline $\begin{array}{l}\text { Subscapular skinfold } \\
\text { (mm) }\end{array}$ & 8.1 (SD 1.6) & 8.2 (SD 1.7) & 8.2 (SD 1.7) & 7.7 (SD 2.0) & 7.5 (SD 1.7) & 7.6 (SD 1.9) \\
\hline $\begin{array}{l}\text { Hemoglobin at } 6 \text { months } \\
(\mathrm{g} / \mathrm{L})\end{array}$ & 107 (SD 12) & 108 (SD 14) & 108 (SD 13) & 109 (SD 11) & 106 (SD 12) & 108 (SD 12) \\
\hline \multicolumn{7}{|l|}{ Breastfeeding duration: } \\
\hline never & $1(0.5 \%)$ & $0(0 \%)$ & $1(0.3 \%)$ & $18(27 \%)$ & $13(22 \%)$ & $31(25 \%)$ \\
\hline$<6$ months & $7(4 \%)$ & $4(2 \%)$ & $11(3 \%)$ & 23 (35\%) & $19(32 \%)$ & $42(34 \%)$ \\
\hline $\begin{array}{r}\text { breastfeeding at } \\
\text { recruitment }\end{array}$ & $182(96 \%)$ & $320(98 \%)$ & $370(97 \%)$ & $25(38 \%)$ & 27 (46\%) & $52(42 \%)$ \\
\hline
\end{tabular}

${ }^{1}$ Significant differences between diet groups among children of HIV-positive mothers: maternal education $(p=0.02)$ and weight at $6 \mathrm{~m}$ ( $\left.p=0.09\right)$.

${ }^{2}$ Significant differences between maternal HIV groups: maternal age $(p<0.001)$, education $(p=0.006)$, occupation $(p=0.04)$, SES ( $\left.p=0.03\right)$, marital status $(p=0.05)$, all anthropometric indicators at $6 \mathrm{~m}(p=0.06$ for triceps; $p \leq 0.003$ for all others), breastfeeding duration $(p<0.001)$. 
Table 2. Effects of treatment arm and maternal HIV status on growth and body composition $Z$ scores during follow-up, in HIV-negative children completing at 18 months HIV-negative children completing at 18 months

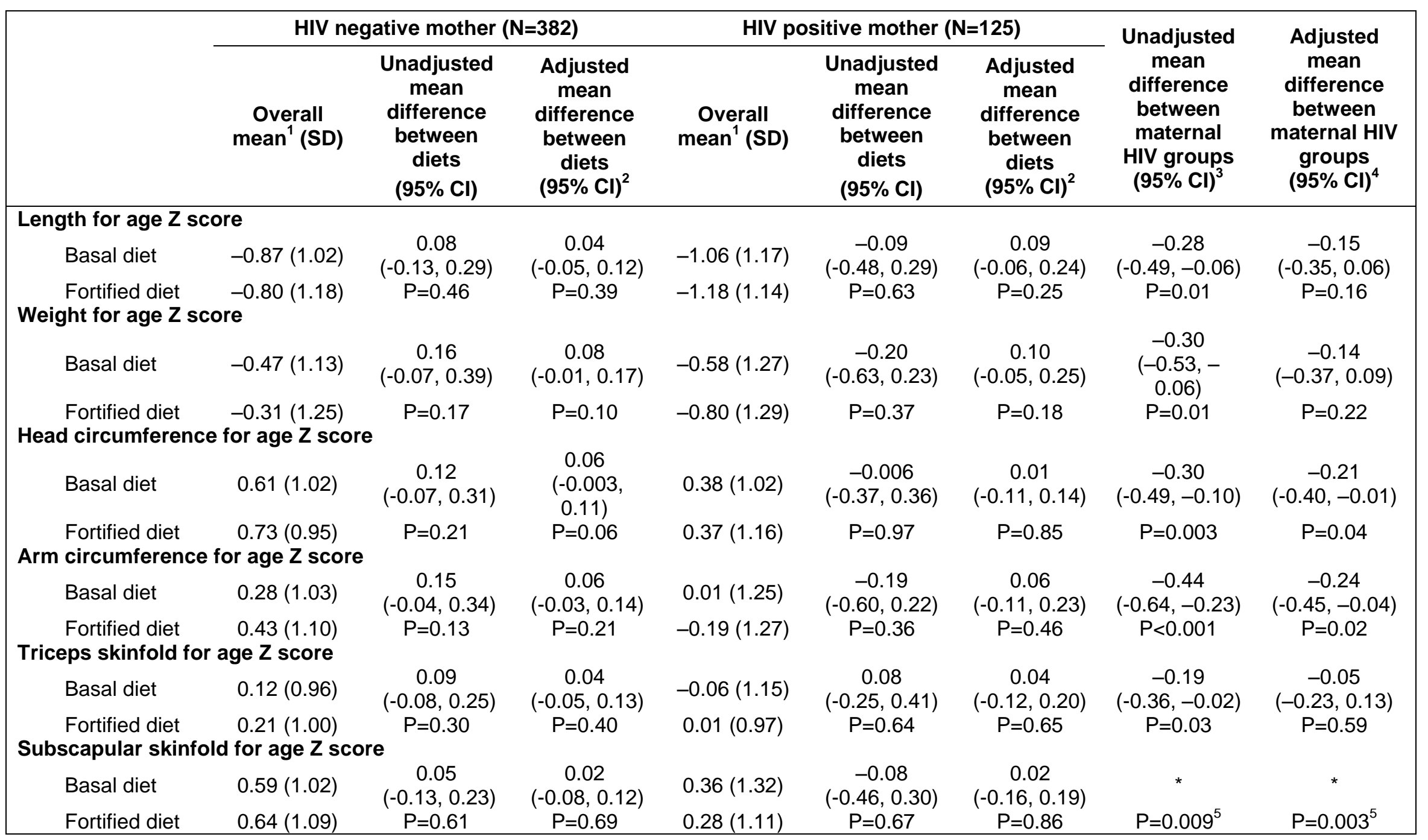


${ }^{1}$ Based on data from 6-18 month visits combined and using random effects regression to account for the correlation of repeated measurements within children. For HIV-negative mothers, from 190 children on basal diet and 192 on fortified diet; for HIV-positive mothers, from 66 children on basal diet and 59 on fortified diet.

${ }^{2}$ Adjusted for visit, SES, maternal education, current breastfeeding, sex and Z-score at 6 months, from repeated-measures randomeffects regression.

${ }^{3}$ Adjusted for diet treatment group and visit month, from repeated-measures random-effects regression.

${ }^{4}$ Adjusted for diet treatment group, visit, SES, maternal education, current breastfeeding and sex, from repeated-measures randomeffects regression

${ }^{5} \mathrm{P}$-value for interaction; unadjusted difference at $6 \mathrm{~m}$ is $-0.45(95 \% \mathrm{Cl}-0.67$ to -0.22$)$; at $12 \mathrm{~m}$ is $-0.27(95 \% \mathrm{Cl}-0.49$ to -0.05$)$; and at $18 \mathrm{~m}$ is $-0.15(95 \% \mathrm{Cl}-0.37$ to 0.07$)$. Adjusted difference at $6 \mathrm{~m}$ is $-0.36(95 \% \mathrm{Cl}-0.06$ to -0.13$)$; at $12 \mathrm{~m}$ is $-0.18(95 \% \mathrm{Cl}-0.42$ to $0.06)$; and at $18 \mathrm{~m}$ is $-0.08(95 \% \mathrm{Cl}-0.30$ to 0.15$)$. 
Table 3. Effects of concurrent breastfeeding on growth and body composition Z scores during follow-up, in HIV-negative children completing at 18 months

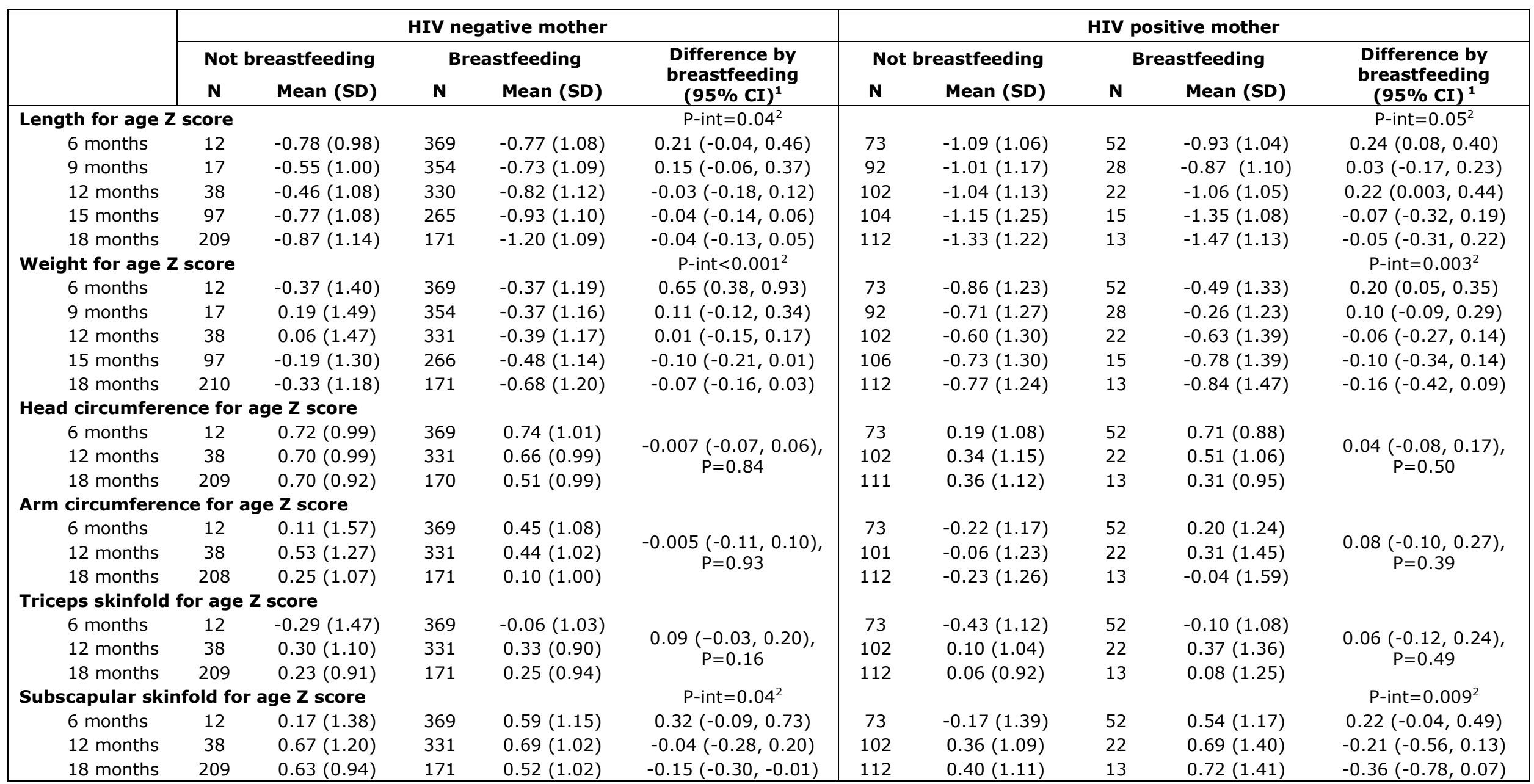

${ }^{1}$ Adjusted for diet group, visit, socioeconomic status, maternal education, sex and baseline Z score, from repeated-measures random-effects regression

${ }^{2}$ Significant interaction between current breastfeeding \& time (visit) - i.e. the effect of breastfeeding on growth varies over time 


\section{Figure legends}

Figure 1. Flow diagram of participants through the study

Sixty-eight children were inadequately randomised because one porridge supply ran out; these children were excluded from analyses.

Figure 2. Anthropometric Z scores of HIV-exposed, uninfected and HIV-unexposed children

Numbers of children were 190 HIV-unexposed in the basal diet group, 192 HIV-unexposed in the richly-fortified diet group, 66 HIV-EU in the basal diet group, and 59 HIV-EU in the richlyfortified group. 